INEEL/CON-98-01179

PREPRINT

\title{
Direct Imaging of Anisotropic Material Properties Using Photorefractive Laser Ultrasound
}

\author{
K. L. Telschow \\ V. A. Deason \\ R. S. Schley \\ S. M. Watson
}

June 28, 1999 - July 2, 1999

\section{$9^{\text {th }}$ International Symposium on Nondestructive Characterization of Materials}

This is a preprint of a paper intended for publication in a journal or proceedings. Since changes may be made before publication, this preprint should not be cited or reproduced without permission of the author.

This document was prepared as a account of work sponsored by an agency of the United States Government. Neither the United States Government nor any agency thereof, or any of their employees, makes any warranty, expressed or implied, or assumes any legal liability or responsibility for any third party's use, or the results of such use, of any information, apparatus, product or process disclosed in this report, or represents that its use by such third party would not infringe privately owned rights. The views expressed in this paper are not necessarily those of the U.S. Government or the sponsoring agency. 


\title{
Direct Imaging of Anisotropic Material Properties using Photorefractive Laser Ultrasound
}

\author{
K.L. Telschow, V. A. Deason, R. S. Schley and S. M. Watson
}

Idaho National Engineering and Environmental Laboratory

Lockheed Martin Idaho Technologies Co.

Idaho Falls, ID 83415-2209

\begin{abstract}
Anisotropic properties of materials can be determined by measuring the propagation of elastic waves in different directions. A laser imaging approach is presented that utilizes the adaptive property of photorefractive materials to produce a real-time measurement of the antisymmetric Lamb or flexural traveling wave mode displacement and phase. Continuous excitation is employed and the data is recorded and displayed in all directions simultaneously at video camera frame rates. Fourier transform of the data produces an image of the wave slowness in all planar directions. The results demonstrate imaging of microstructural isotropy and anisotropy and stress induced ansiotropy in plates.
\end{abstract}

\section{INTRODUCTION}

A powerful method for imaging ultrasonic motion has been developed at the INEEL that utilizes the photorefractive effect in optically nonlinear materials to perform adaptive interferometry. ${ }^{1,2}$ Utilizing this approach, no postprocessing of the data recorded by a video camera is required to produce images of the surface vibration amplitude over large areas. This paper describes application of this approach, referred to as the INEEL Laser Ultrasonic Camera, to imaging of traveling antisymmetric Lamb or flexural waves in plates for determining anisotropic elastic properties of materials produced by microstructural texture and by the external application of stress. Resonant motion in plates has been previously described. ${ }^{3}$

Optical interference is developed within a photorefractive material with this technique and the output is an optical image whose intensity distribution is directly proportional to the surface vibration amplitude, for small ultrasonic displacements. In certain nonlinear optical materials, the photorefractive process can be established, whereby, optical excitation and transport of charge carriers produces a diffraction grating or hologram from an optical interference pattern. A charge distribution results spatially and temporally in the material that is determined by the phase information 
impressed onto optical signal and reference beams (e.g. by a vibrating surface). The INEEL method records the photorefractive grating produced at a fixed beat frequency between the phase modulated signal and reference beams. The response is proportional to the Bessel function of order one, providing a linear output intensity signal for small ultrasonic amplitudes. The method accommodates rough surfaces, exhibits a flat frequency response above the photorefractive response cutoff frequency, and can be used for detecting both standing and traveling waves.

\section{EXPERIMENTAL METHOD}

The experimental schematic for ultrasonic detection is shown in Figure 1. A solid state laser source at $\lambda=532 \mathrm{~nm}$ provided the signal and reference beams. The traveling wave motion occurring on the plate surface (driven at its center by a continuously excited piezoelectric transducer) produced a phase modulation at frequency and phase $\omega_{s}, \varphi_{s}$ on the signal beam. The reference beam was phase modulated by an electrooptic modulator at a fixed modulation amplitude at frequency and phase $\omega_{r}, \varphi_{r}$. The modulated beams were combined and interfered inside a Bismuth Silicon Oxide (BSO) photorefractive crystal with operation in the diffusive regime. In the four-wave mixing configuration, the reference beam was reflected back into the crystal along a counterpropagating path to produce an output phase conjugate beam. The vibration modulated phase grating was read out by a video camera recording the resulting diffacted beam that propagated backward along the signal beam leg and deflected with a beamsplitter, as shown. This four-wave mixing configuration provides separation between the demodulated ultrasonic motion image and the visual image of the plate surface.

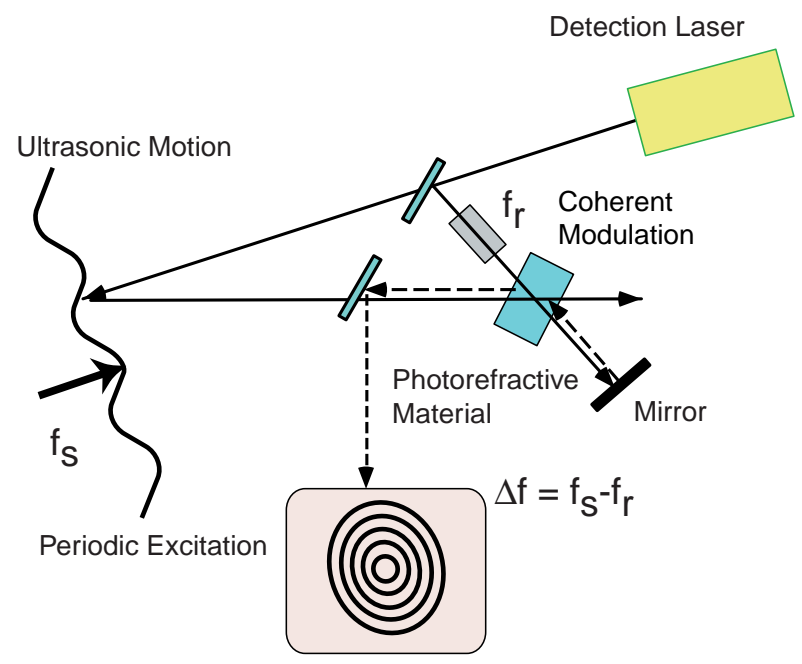

FIGURE 1. Schematic of the INEEL Laser Ultrasonic Camera. 


\section{FLEXURAL WAVE IMAGING ON PLATES}

Flexural wave motion in a plate, with displacement normal to the plate surface, generated from an oscillating point excitation force $F_{0}$, at frequency and phase $\omega_{s}, \varphi_{s}$, for ultrasonic wavelengths $\lambda_{a}$ larger than the plate thickness, is given by ${ }^{4}$

$$
\xi(\rho, t)=\operatorname{Re}\left(i \xi_{0}\left[H_{0}^{1}\left(k_{a} \rho\right)-H_{0}^{1}\left(i k_{a} \rho\right)\right] e^{-i\left(\omega_{s} t+\varphi_{s}\right)}\right)
$$

where $\xi_{0} \equiv \frac{F_{0}}{8 \omega \sqrt{D \sigma}}, \quad k_{a}=\frac{2 \pi}{\lambda_{a}}$ and $H_{0}^{1}(x)$ is the Hankel function of order zero representing a wave traveling outward from the origin and $\rho$ is the radial coordinate. $D \equiv \frac{E h^{3}}{12\left(1-s^{2}\right)}$ is the bending stiffness of the plate, $\sigma \equiv \rho_{m} h$ the mass density per unit area, $\rho_{m}=$ the mass density, $s=$ Poisson's ratio, $E=$ Young's modulus and $h=$ the plate thickness. The two dimensional spatial Fourier transform is ${ }^{5}$

$$
\widetilde{\xi}_{\rho}(q)=\frac{F_{0}}{D}\left[\frac{1}{\left(q^{2}-k_{a}^{2}\right)\left(q^{2}+k_{a}^{2}\right)}\right]
$$

yielding poles at the propagation wavevector $k_{a}^{4} \equiv \frac{\sigma \omega^{2}}{D}$. An image of the ultrasonic wave consists of concentric circles depicting wavefronts. In the transform domain, a single circle is found depicting the wave slowness for this isotropic material. These circles become oblong for anisotropic materials, as is shown below.

The mechanism for image recording is that of optical lock-in operation, whereby the only image recorded is that which is coherent with the reference modulation. ${ }^{6}$ The first time varying or AC term in the intensity of the diffracted beam is given by

$$
I_{4} \propto J_{0}\left(\frac{4 \pi \xi_{0}}{\lambda}\right) J_{1}\left(\frac{4 \pi \xi_{0}}{\lambda}\right)\left[\frac{\cos (\Omega t+\Phi-\chi(\rho)-\psi)}{\sqrt{1+\Omega^{2} \tau^{2}}}\right]
$$

where $\Phi=\varphi_{r}-\varphi_{s}, \Omega=\omega_{r}-\omega_{s}, \tau$ is the photorefractive material time constant, $\tan (\psi)=\Omega \tau$, and $\chi(\rho)=\left(k_{a} \rho-\frac{\pi}{4}+\Phi_{a}\right)$ is the ultrasonic wave phase with respect to the source. The resultant intensity is proportional to the ultrasonic wave amplitude for small displacements relative to the optical wavelength, $\left(\frac{4 \pi \xi(\rho, t)}{\lambda}<<1\right)$. 


\section{ULTRASONIC WAVE IMAGING}

\section{Isotropic Materials}

Figure 2 shows the camera image of a traveling flexural wave in an isotropic nickel plate at $30 \mathrm{kHz}$ with $h=0.125 \mathrm{~mm}, E=204 \mathrm{GPa}, \mathrm{s}=0.31, \rho_{m}=8.9 \mathrm{~g} / \mathrm{cm}^{3}$. The expected circular wavefronts due to the isotropic microstructure of the nickel plate are clearly defined. The figure shows single frame image data. The photorefractive process yields a true picture of the actual wave vertical displacement motion and requires no additional processing to generate the spatial images shown. The magnitude of the Fourier transform of the traveling wave displacement image is a single ring at the wavevector delineating the propagating mode that quantitatively determines the elastic constants and the isotropic character of the plate. This analysis procedure provides considerable information about the plate in one simple image.

\section{Anisotropic Materials}

If the specimen is elastically anisotropic, then the wave speed varies with the propagation direction. Many materials, produced in sheet form, have orthotropic elastic symmetry. Figure 3 shows this type of behavior for traveling waves in a sheet of $42 \mathrm{lb}$. linerboard paper ${ }^{7,8}$. The fibers of the paper sheet were aligned approximately along the vertical direction. The oblong patterns for both the wavefronts and the wave slowness show the anisotropy immediately.

Another example is illustrated by measurements on a brass sheet under stress. The effect of uniaxial stress alters the flexural wave speed in a manner described by linear elasticity theory. In particular, an isotropic material becomes anisotropic with a flexural wave speed that increases with stress along the principal stress direction. The effect is very pronounced for the flexural mode as the wave speed vanishes at zero frequency in the absence of stress but not if stress is present.
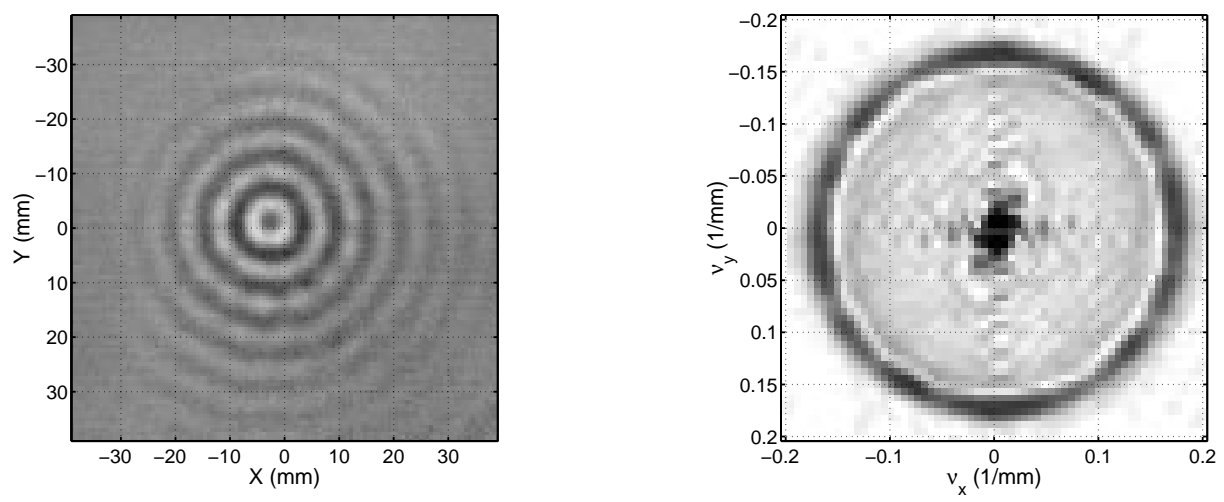

Figure 2. Ultrasonic Wave Image in a Nickel Plate and the Fourier Transform of the Camera Image. 

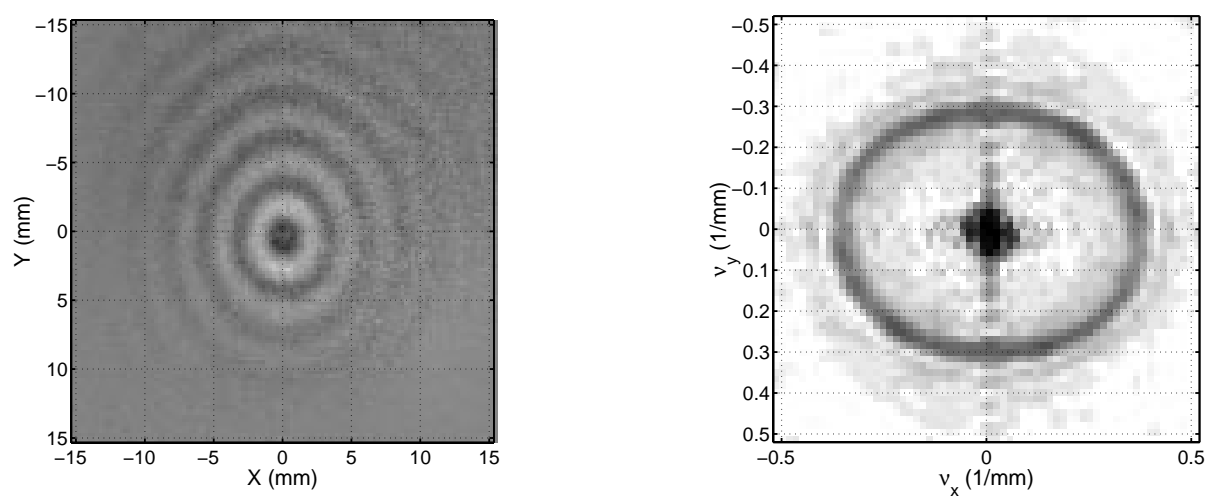

Figure 3. Ultrasonic Wave Image in a Paper Sheet and the Fourier Transform of the Camera Image.

Figure 4 shows measurements on a nominally 0.002 " thick sheet of brass in the "as produced" condition and with various amounts of externally applied uniaxial stress. Even the "as produced" sheet shows an anisotropy equivalent to an applied stress of about $47 \mathrm{MPa}$. Calculations of the uniaxial stress dependence of the wave slowness in all directions were performed using the isotropic elastic properties for brass of $E=103 \mathrm{GPa}, s=0.38, \rho_{m}=8.5 \mathrm{~g} / \mathrm{cm}^{3}$. Imaging measurements were performed under stress in the same direction as the perceived residual stress. This one measurement is not sufficient to determine if the plate had this residual stress or the

(a)
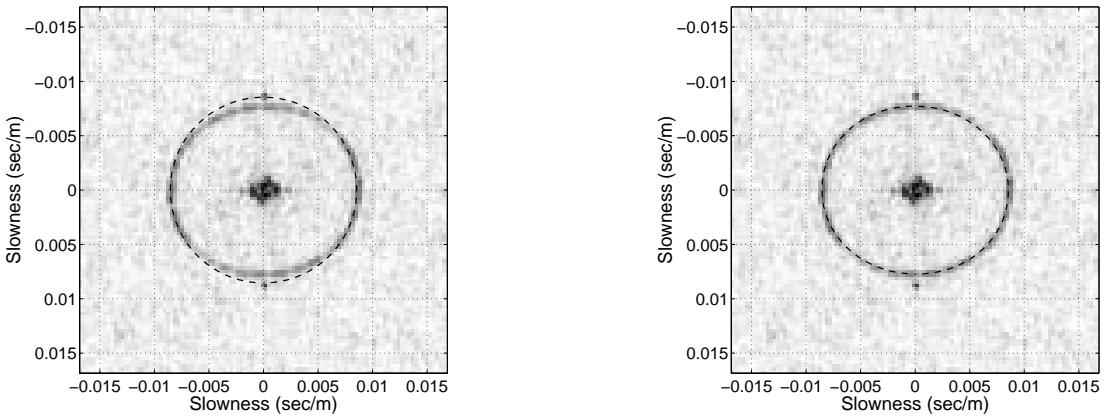

(c)
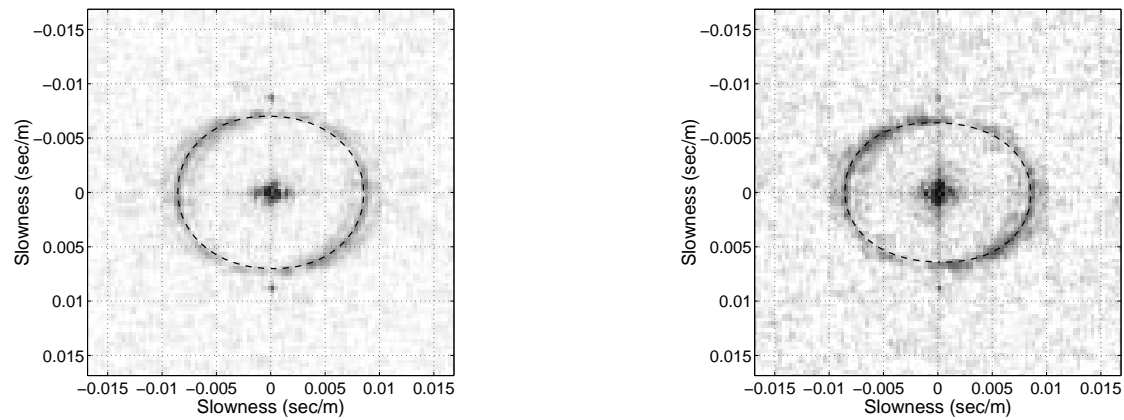

Figure 4. Fourier transform wave slowness diagrams for a brass plate under externally applied uniaxial stress (a) 0, (b) $0 \mathrm{MPa}$, (c) $47 \mathrm{MPa}$, (d) $95 \mathrm{MPa}$. The dashed line in (a) assumes isotropic conditions and in (b)-(d) is calculated using a residual stress of $47 \mathrm{MPa}$ in addition to the indicated applied stress. 
anisotropy was due to inherent microstructural texture introduced by the rolling process. However, the results show good agreement between the experimental measurements and the calculations for external stresses up to $95 \mathrm{MPa}$.

\section{CONCLUSIONS}

An imaging laser ultrasonic camera has been described. Direct two-dimensional surface images of traveling waves on isotropic and anisotropic plates were obtained. These images show the ultrasonic wavefront shape in all planar directions simultaneously thereby illustrating anisotropic elastic behavior directly. Fourier transform of these wavefront images produces a wave slowness diagram yielding a quantitative method for obtaining the elastic stiffness anisotropy of sheet materials. Coupled with detailed modeling of the elastic wave modes, the INEEL Laser Ultrasonic Camera provides an important capability for determining anisotropy in materials by using data in all planar directions simultaneously.

\section{ACKNOWLEDGMENTS}

This work was sponsored by the U.S. Department of Energy, Office of Energy Research, Office of Basic Energy Sciences, Engineering Research program under DOE Idaho Operations Office Contract DE-AC07-94ID13223.

\section{REFERENCES}

P. Yeh, Introduction to Photorefractive Nonlinear Optics, (John Wiley, New York, 1993).

S. I. Stepanov, International Trends in Optics, (Academic Press, New York, 1991) Ch. 9.

T. C. Hale, K. L. Telschow, and V. A. Deason, Applied Optics, 111, 8,248-8,258 (1997).

P. M. Morse and K. U. Ingard, Theoretical Acoustics, (McGraw-Hill, New York, 1968) 219.

$5 \quad$ K.L. Telschow, V. A. Deason, R. S. Schley and S. M. Watson, "Direct Imaging of Lamb Waves in Plates using Photorefractive Dynamic Holography," to be published.

6 K. L. Telschow, V. A. Deason, R. S. Schley and S. M. Watson, Review of Progress in QNDE, Vol. 18, Eds. D.O. Thompson and D. E. Chimenti, (Plenum Press, New York, 1999) 999-1005.

7 The paper sample was provided by the Institute of Paper Science and Technology, Atlanta, Georgia.

8 K.L. Telschow, V. A. Deason, J. B. Walter, R. S. Schley and S. M. Watson, INEEL External Report INEEL/EXT-98-00920, January 1999. 